\title{
Computer simulations of catanionic surfactants adsorbed at air/water interfaces. II. Full coverage
}

\author{
Esteban Clavero \\ Departamento de Química Inorgánica, Analítica y Química-Física, Facultad de Ciencias Exactas y \\ Naturales, Universidad de Buenos Aires, Ciudad Universitaria, Pabellón II, 1428 Buenos Aires, Argentina \\ and INQUIMAE, Facultad de Ciencias Exactas y Naturales, Universidad de Buenos Aires, Ciudad \\ Universitaria, Pabellón II, 1428 Buenos Aires, Argentina \\ Javier Rodriguez and Daniel Laria ${ }^{\text {a) }}$ \\ Departamento de Química Inorgánica, Analítica y Química-Física, Facultad de Ciencias Exactas y \\ Naturales, Universidad de Buenos Aires, Ciudad Universitaria, Pabellón II, 1428 Buenos Aires, Argentina, \\ INQUIMAE, Facultad de Ciencias Exactas y Naturales, Universidad de Buenos Aires, Ciudad \\ Universitaria, Pabellón II, 1428 Buenos Aires, Argentina and Unidad Actividad Física, Comisión Nacional \\ de Energía Atómica, Avenida Libertador 8250, 1429 Buenos Aires, Argentina
}

(Received 2 July 2007; accepted 14 August 2007; published online 27 September 2007)

\begin{abstract}
We extend our previous molecular dynamics experiments [Rodriguez et al., J. Phys. Chem. B 109, 24427 (2005)] to the analysis of the adsorption of catanionic surfactants at water/air interfaces, at a surfactant coverage close to that of the saturated monolayer: $30.3 \AA^{2}$ per headgroup. The mixture of surfactants investigated corresponds to equal amounts of dodecytrimethylammonium (DTA) and dodecylsulfate (DS). The structure of the interface is analyzed in terms of the local densities and orientational correlations of all relevant interfacial species. In accordance with experimental evidence, the DTA headgroups penetrate deeper into the aqueous substrate than the DS ones, although the average positions of all headgroups, with respect to the interface, lie in positions somewhat more external than the ones observed at lower coverages. Average tail tilts are close to $45^{\circ}$. The characteristics of the headgroup-water substrate correlations are also analyzed using a tessellation procedure of the interface. The density and polarization responses of the interfacial domains closest to the DS headgroups are enhanced, compared to those adjacent to the DTA detergents. Dynamical aspects related to the diffusion and to the orientational correlations of different water layers in close contact with the surfactant are also investigated. () 2007 American Institute of Physics. [DOI: 10.1063/1.2779876]
\end{abstract}

\section{INTRODUCTION}

Many technological and practical applications of tensoactive substances make use of the particular physicochemical properties exhibited by surfactant mixtures. ${ }^{1}$ In particular, blends comprising anionic and cationic surfactants lead to peculiar combinations, normally referred to as catanionic solutions. ${ }^{2}$ Depending on the individual concentrations of each component, these systems may present a rich variety of strongly structured phases. Among others, the list includes micelles, vesicles, swollen lamellar phases, and flat nanodisks, whose linear dimensions may extend up to several hundreds of nanometers. ${ }^{3-7}$ All these self-assembled structures exhibit properties well differentiated from those observed in much simpler, i.e., one component, surfactant solutions. These distinctive features, in turn, are manifested in larger absorptions, unusually strong surface activity, and shifts in the critical micellar concentrations, to quote just a few important examples. ${ }^{8,9}$ At a microscopic level, the origins of this synergism is usually ascribed to the strong Coulomb coupling that prevails in the interactions between the oppositely charged headgroups.

\footnotetext{
${ }^{a)}$ Author to whom correspondence should be addressed. Electronic mail: dhlaria@cnea.gov.ar
}

Concerning the behavior of these mixtures acting as coating films at air/water interfaces, a molecular interpretation based on the sole consideration of the Coulomb interactions is somewhat incomplete. There is a large body of experimental information available ${ }^{10-15}$ that shows that the dynamic and equilibrium properties of the resulting interfaces are also very much dependent on the branching and chain lengths of the hydrophobic segments of the individual surfactants. In contrast, from the theoretical side, the information available on these complex mixtures is not that abundant and have mainly focused on predictions of their thermodynamic properties based on classical theories of liquid mixtures. ${ }^{16-19}$ In addition, a few computer simulation studies have also been undertaken, ${ }^{20-22}$ although the systems investigated included anionic/nonionic mixtures adsorbed at air/ water and liquid/liquid interfaces exclusively. Using molecular dynamics techniques, we recently performed a first exploratory study ${ }^{23}$ of the structural features of one of the simplest catanionic surfactant mixtures comprising single chained compounds: an equimolar mixture of dodecylsulfate (DS) and dodecyltrimethyammonium (DTA). In that work, we analyzed the nature of the surface solvation of single surfactant pairs and the adsorption of surfactant films at intermediate coverages as well. One of the most important 
conclusions that we could draw from this preliminary study is that the gross features of the structure of the surfactants at low coverages can be described in terms of a partially screened, two-dimensional, simple Coulomb fluid. In an effort to provide a more comprehensive description of the behavior of these insoluble monolayers over the whole surface concentration range, the present work complements the previous one and focuses on equilibrium and dynamical aspects pertaining to the adsorbed film and the underlying water substrate.

The organization of the work is as follows. Details of the simulation procedure and model are briefly described in Sec. II. The equilibrium results of the work are presented in Sec. III, while Sec. IV contains information about the dynamics of the interface. Finally, the main conclusions are presented in Sec. V.

\section{MODEL AND SIMULATION PROCEDURE}

The model and simulation procedure adopted to perform the molecular dynamics experiments were similar to the ones described in our previous paper. ${ }^{23}$ So, for the sake of concision, we will present here only a brief overview of the main details and refer the interested reader to Ref. 23 for a more comprehensive presentation.

Simulation experiments began by distributing equal amounts, $N_{s}=13$, of DS $\left[\mathrm{CH}_{3}\left(\mathrm{CH}_{2}\right)_{11} \mathrm{OSO}_{3}\right]^{-}$and DTA $\left[\mathrm{CH}_{3}\left(\mathrm{CH}_{2}\right)_{11} \mathrm{~N}\left(\mathrm{CH}_{3}\right)_{3}\right]^{+}$ions on top of one of the two air/ water interfaces of a previously equilibrated aqueous slab, containing $N_{w}=648$ water molecules. This substrate was originally generated from a fully periodic system of volume $28.06 \times 28.6 \times 24.95 \AA^{3}$, in which periodic boundary conditions were suppressed along the shortest axis of the simulation box (hereafter referred to as the $z$ axis). With these dimensions, the resulting area per surfactant headgroup was $\rho_{s}^{-1}=30.3 \AA^{2}$. This degree of coverage is approximately twice the one investigated in our previous study ${ }^{23}$ and corresponds to the estimated value of the saturated monolayer reported in Ref. 13.

The dynamical trajectories corresponded to microcanonical runs at temperatures close to $T \approx 298 \mathrm{~K}$. At this temperature regime, the interfaces were stable and no significant evaporation was observed. All simulation runs were preceded by a second thermalization period of $\sim 0.2 \mathrm{~ns}$, during which only the surfactant tails were allowed to move at $T$ $\sim 700 \mathrm{~K}$; after that, the systems were gradually cooled down to ambient temperature, during a subsequent time interval of 0.2 ns. Statistics were collected during equilibrium trajectories typically lasting $\sim 5 \mathrm{~ns}$. Full details of the force fields and treatment of long-ranged forces can be found in Ref. 23.

\section{STRUCTURE AND POLARIZATION}

\section{A. Surfactant monolayer and water substrate}

The starting point of our analysis will be the consideration of the local densities of the different components which are present at the surfactant coated interface of the slab. For the water case, we focused our attention on the number of water molecules per unit of area $A$ at the $x y$ plane, with their oxygen sites lying between $z$ and $z+d z$,

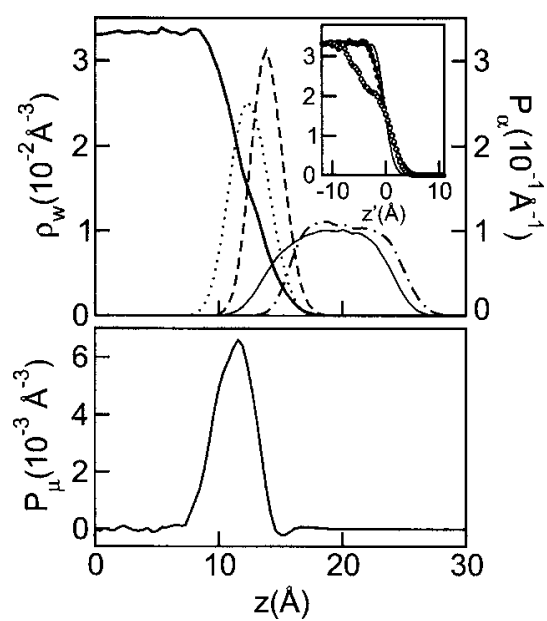

FIG. 1. Top panel: water local density (thick solid lines, left axis) and probability densities (right axis) for the surfactant groups. DTA head and tail groups are shown with dotted and thin solid lines, while DS head and tail groups are shown with dashed and dot-dashed lines. The inset contains results for the bare interface (black circles) and for intermediate surfactant coverages (open circles) taken from Ref. 23 ( $z^{\prime}$ coordinates represent distances to the corresponding Gibbs dividing surfaces). Bottom panel: polarization density of the water slab.

$$
\rho_{w}(z) d z=\frac{1}{A}\left\langle\sum_{i=1}^{N_{w}} \delta\left(Z_{i}-Z_{\text {c.m. }}-z\right)\right\rangle d z,
$$

where $\langle\cdots\rangle$ denotes an equilibrium ensemble average and $Z_{i}$ and $Z_{\mathrm{c} . \mathrm{m} \text {. }}$ represent the $z$ coordinates of the $i$ th water oxygen and the center of mass of the water slab, respectively. The plot of $\rho_{w}(z)$ is shown in top panel of Fig. 1. Two regions are clearly perceptible: a central portion of the slab, near $z \sim 0$, where the density is rather uniform and close to the usual bulk water value $\left(\rho_{b}=0.033 \AA^{-3}\right)$. In addition, one also observes a lateral interface, where the decay in density takes place over a lengthscale of a few angstroms. The latter profile can be reasonably well described by mean-field predictions, namely, ${ }^{24}$

$$
\rho_{w}(z) \sim \frac{\rho_{b}}{2}\left[1-\tanh \frac{z-\bar{z}_{w}}{\Delta z_{w}}\right] .
$$

Nonlinear fits of the decay yield an interface width $\Delta z_{w}$ $=2.6 \AA$, a value that is approximately twice the one of the bare interface $\left(\Delta z_{w} \sim 1.4 \AA\right){ }^{23}$

Surface spatial correlations involving different surfactant segments were analyzed using correlation functions involving the ionic headgroups (HD) and the methylene groups in the hydrophobic tails (TL). More specifically, for each surfactant species, we computed

$$
P_{\alpha} \propto\left\langle\sum_{i} \delta\left(z_{i}^{\alpha}-Z_{\text {c.m. }}-z\right)\right\rangle .
$$

In the previous equation, $z_{i}^{\alpha}$ denotes the $z$ coordinate of the $\alpha$ site in the $i$ th surfactant $(\alpha=\mathrm{HD}, \mathrm{TL})$. For head contributions, we included positions of all sites embodied in the $-\mathrm{SO}_{3}^{-}$and the $-\mathrm{N}\left(\mathrm{CH}_{3}\right)_{3}^{+}$groups; for tail correlations, we considered the positions of the $11 \mathrm{CH}_{2}$ sites and that of the distal $\mathrm{CH}_{3}$ group as well. Headgroup distributions are also shown in Fig. 1 and exhibit Gaussian profiles of the type 
TABLE I. Solvation parameters for superficial solvation of catanionic surfactants at different surface coverages.

\begin{tabular}{lccccccc}
\hline \hline & & & & & & & \\
& $N_{s}$ & $(\AA)$ & $\begin{array}{l}\delta_{\mathrm{TL}} \\
(\AA)\end{array}$ & $\begin{array}{c}\sigma_{\mathrm{HD}} \\
(\AA)\end{array}$ & $\begin{array}{l}\sigma_{\mathrm{TL}} \\
(\AA)\end{array}$ & $\left\langle\cos \theta_{\mathrm{tl}}\right\rangle$ & $\begin{array}{c}\left\langle r_{\mathrm{tl}}\right\rangle \\
(\AA)\end{array}$ \\
\hline \multirow{2}{*}{$\mathrm{AS}$} & 13 & 1.7 & 8.0 & 3.7 & 9.5 & 0.71 & 15.1 \\
& $5^{\mathrm{a}}$ & -2.6 & 3.0 & 5.2 & 8.0 & 0.55 & 13.2 \\
& 13 & 0.2 & 6.8 & 4.5 & 10.4 & 0.72 & 14.1 \\
& $5^{\mathrm{D}}$ & -3.6 & 2.7 & 5.2 & 10.0 & 0.70 & 11.0 \\
\hline
\end{tabular}

${ }^{\mathrm{a}}$ Taken from Ref. 23.

$$
P_{\alpha}(z) \sim\left(\frac{4}{\pi \sigma_{\alpha}^{2}}\right)^{1 / 2} \exp \left[-\frac{4\left(z-\bar{z}_{\alpha}\right)^{2}}{\sigma_{\alpha}^{2}}\right]
$$

Estimates for the different group positions relative to the location of the interface can be readily expressed in terms of $\delta_{\alpha}=\bar{z}_{\alpha}-\bar{z}_{w}$. Results for $\delta_{\alpha}$ appear in Table I; similarly to what was reported at lower coverages ${ }^{23}$ and in accordance with experimental evidences, ${ }^{25,26}$ cationic surfactant headgroups "plunge" deeper into the aqueous substrate than the headgroups of the anionic surfactant. Anyhow, the extent of the overall penetration is not significant and much less marked than the one observed at intermediate surface coverages. Note that the cationic headgroups practically lie at the Gibbs dividing surface, while the anionic groups are located $1 \AA$ outward from this position. In addition, anionic and cationic headgroup distributions show narrower widths (expressed in terms of $\sigma_{\mathrm{HD}}$ ) at higher surface coverages (see entries in Table I). More interesting is the fact that the resulting fluctuations in the local density of the aqueous substrate look much less pronounced at high surfactant coverages than at intermediate ones. In the inset of Fig. 1, we show zoomed plots for the density profiles at the vicinity of the Gibbs dividing surfaces of bare, mid-, and full-covered interfaces. Clearly, deviations from mean-field predictions do not follow a monotonous surfactant density trend and are much more marked in the midcoverage regime.

Tail distributions are accordingly displaced to the head shifts described in the previous paragraph and look similarexcept perhaps for a somewhat "flatter" central region-to their headgroup counterparts. Although tail locations with respect to the interface do not considerably charge with the degree of coverage, we did observe clear distinctions in their overall structures. In Fig. 2, we present results for the tilt distributions,

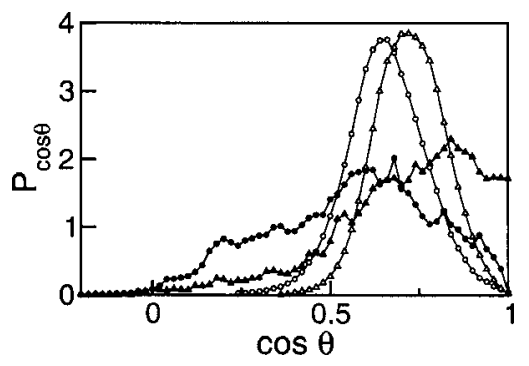

FIG. 2. Tilt distributions of catanionic mixtures of surfactants at $\rho_{s}^{-1}$ $=30.3 \AA^{2}$ (white symbols) and $\rho_{s}^{-1}=78.7 \AA^{2}$ (black symbols). DS (DTA) data are shown in circles (triangles).
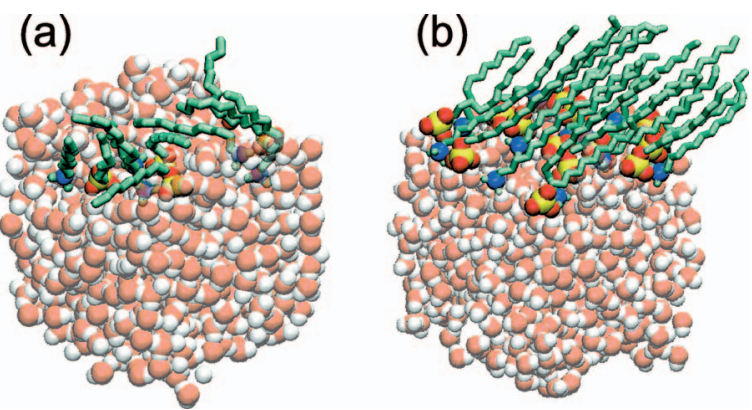

FIG. 3. (Color) Snapshots for typical configurations of catanionic mixtures adsorbed at water/air interfaces with different surface coverages: (a) $\rho_{s}^{-1}$ $=78.7 \AA^{2}$ and (b) $\rho_{s}^{-1}=30.3 \AA^{2}$.

$$
P_{\cos \theta_{\mathrm{tl}}}=\left\langle\delta\left(\cos \theta-\cos \theta^{\prime}\right)\right\rangle,
$$

where

$$
\cos \theta^{\prime}=\frac{\mathbf{r}_{\mathrm{tl}} \cdot \hat{\mathbf{z}}}{\left|\mathbf{r}_{\mathrm{t} 1}\right|}
$$

In the previous equation, $\mathbf{r}_{\mathrm{tl}}=\mathbf{r}_{\mathrm{CH}_{3}}-\mathbf{r}_{\mathrm{CH}_{2}}$ is the tail vector joining the methylene group closest to the head and the distal methyl group, while $\hat{\mathbf{z}}$ is a unit vector along the $z$ direction. Averages over these distributions are also presented in Table I $\left(\left\langle\cos \theta_{\mathrm{t} 1}\right\rangle\right)$. Although the latter values are still comparable, a direct inspection of the plots reveals that packing effects promote more localized distributions at high coverages. Moreover, there is a clear change in the prevailing interactions determining the overall structure of the surfactants: at low coverages, tail-water substrate interactions seem to be the relevant contributions to the interfacial solvation of the detergents, while at high coverages, tail-tail interactions dominate the energetics of the solvation (not shown). Figure 3 includes two snapshots of instantaneous configurations of the interface at intermediate and full coverage regimes where these distinctive features are self-evident.

We also analyzed the local polarization of the interface as described by the following density.

$$
P_{\mu}(z)=\frac{1}{A}\left\langle\sum_{i=1}^{N_{w}} \cos \theta_{i} \delta\left(Z_{i}-Z_{\mathrm{c} . \mathrm{m} .}-z\right)\right\rangle,
$$

where

$$
\cos \theta_{i}=\frac{\boldsymbol{\mu}_{i} \cdot \hat{\mathbf{z}}}{\left|\boldsymbol{\mu}_{i}\right|}
$$

In the previous equation, $\boldsymbol{\mu}_{i}$ represents the dipole moment of the $i$ th water molecule. The results are shown in the bottom panel of Fig. 1. Note that, although there is no net charge at the interface, the presence of the surfactant mixture still promotes a net dipolar alignment at the surface. As such, the positive sign in the polarization profile would suggest that the DS headgroups are able to promote a larger extent of alignment of the water substrate than the DTA ones. Yet, the magnitude of the net polarization is rather small; by combining the results of both panels, one can estimate the average 


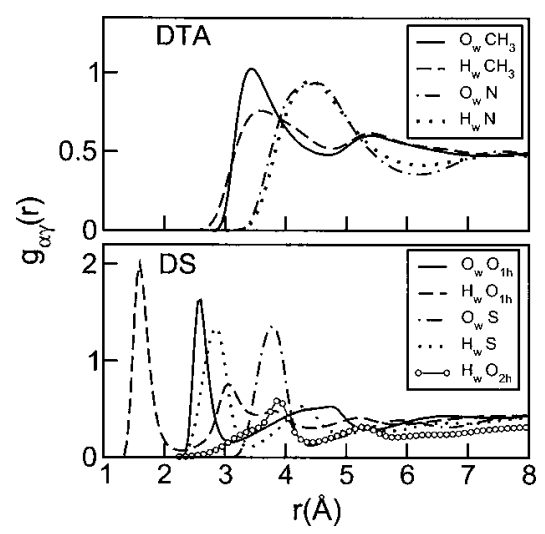

FIG. 4. Pair correlation functions between water and different detergent sites in catanionic mixtures (see text).

value of the individual dipole orientation $\left\langle\theta_{i}\right\rangle \sim 70^{\circ}$ (in passing, we recall that at bare interfaces, ${ }^{27}\left\langle\theta_{i}\right\rangle \sim 90^{\circ}$ ). We further investigated additional aspects related to polarization fluctuations of the interface whose descriptions we prefer to postpone, for the purposes of clarity, to the next section.

\section{B. Water-surfactant correlations}

The sole characterization of the interface structure expressed in terms of the values of $\Delta z_{w}$ is rather incomplete. We pointed out that the average positions of the surfactant headgroups in the mixture are shifted along the $z$ axis, so it is reasonable to infer that this should lead to fluctuations of the overall roughness of the interface over surface "patches" with characteristic lengthscales of the order of $\rho_{s}^{-1 / 2}$ $\sim 5-6 \AA$.

In order to gain a deeper understanding of the characteristics of such fluctuations and of the water structure in the close vicinity of the headgroups, we found it convenient to perform a local analysis based on water-surfactant spatial correlations. In this context, the usual starting point is the consideration of site-site radial distribution functions of the type

$$
g_{\alpha \gamma}(r)=\frac{1}{4 \pi r^{2} \rho_{\alpha} N_{\gamma}}\left\langle\sum_{\gamma}^{N_{\gamma}} \delta\left(\left|\mathbf{r}_{\alpha^{w}}-\mathbf{r}_{\gamma^{\mathrm{HD}}}\right|-r\right)\right\rangle .
$$

In the previous equation, the indices $\alpha$ and $\gamma$ denote sites in water ( $w)$ and surfactant headgroups (HD), respectively, while $\rho_{\alpha}$ represents the density of site $\alpha$ in the bulk ( $\rho_{\mathrm{O}}$ $\left.=0.5 \rho_{\mathrm{H}}=\rho_{b}\right)$. Results for correlations of a few relevant headgroup sites are shown in the top and bottom panels of Fig. 4 . From the direct inspection of the magnitudes of the main peaks, it is clear that DS is able to promote a larger extent of structuration in the neighboring water. A more detailed analysis shows that the surface solvation of the DS is dominated by $\sim 1.7$ hydrogen bonds of the type $\mathrm{H}_{w} \cdots \mathrm{O}_{1 h}$ (here $1 h$ stands for oxygen sites in the $-\mathrm{SO}_{3}$ group). These tightly bound water molecules seem also to be the ones contributing to the main peaks, involving $\mathrm{S}$ and the ether oxygen sites $\left(\mathrm{O}_{2 h}\right)$ in the surfactants, both located at $r \sim 3.8 \AA$. On the other hand, the structure of water surrounding DTA headgroups is characterized by much broader $\mathrm{O}_{w}-\mathrm{CH}_{3}$ and $\mathrm{H}_{w}-\mathrm{CH}_{3}$ peaks, both centered at $3.5 \AA$, including $\sim 6$ and

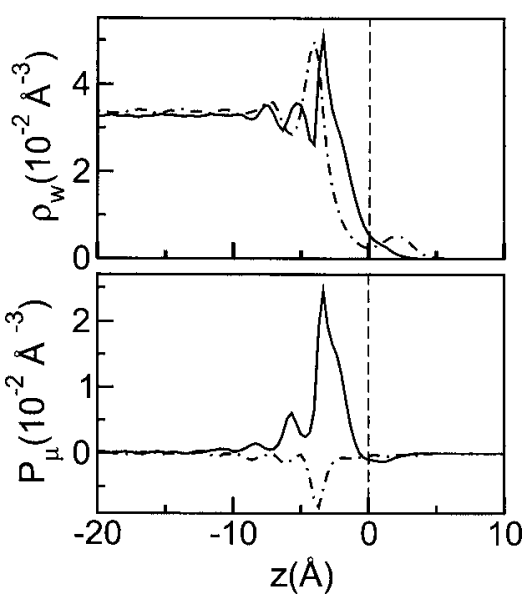

FIG. 5. Top panel: water local density across tessellated substrate domains. Bottom panel: same as top panel for polarization density. Results for DS (DTA) domains are shown with solid (dashed) lines.

$\sim 12$ sites, respectively. In this respect, the structure around the portion of the $\mathrm{CH}_{3}$ groups exposed to the water substrate resembles - at a qualitative level - the one observed in the solvation of simple hydrophobic solutes, such as methane, in bulk water.

The radial correlations shown in Fig. 4, although physically meaningful, do not exhibit the usual asymptotic behavior at large distances corresponding to the homogeneous fluid [i.e., $g_{\alpha \gamma}(\infty) \sim 1$ ] since they do not capture the correct symmetry of the surface environment. One can consider a complementary analysis from a different perspective, which incorporates a more adequate description of the local inhomogeneities prevailing at the interface. The key element of this alternative relies on a tessellation of the water substrate. The main idea of the procedure is conceptually simple and has been thoroughly described in Ref. 28. Briefly, the procedure involved the following steps: (i) $\mathrm{S}$ and $\mathrm{N}$ atoms in all headgroups were brought to an arbitrary plane, by shifting their $z$ coordinates (hereafter denoted as $Z_{\mathrm{HD}}^{\alpha}$ ); (ii) the latter plane was tessellated by assuming that the positions of the headgroups corresponded to centers of Voronoi polygons; (iii) these polygons were considered as sections of "columnar" water domains across the slab, oriented along the $z$ axis. These domains, in turn, allowed an appropriate tagging of the water substrate, with molecules "associated" with either surfactant species.

In the top panel of Fig. 5, we present the profiles of the $z$-dependent water densities along DS and DTA domains,

$$
\rho_{w}^{\alpha}(z)=\frac{1}{A^{\alpha}}\left\langle\sum_{i=1} \delta\left(Z_{i}^{\alpha}-Z_{\mathrm{HD}}^{\alpha}-z\right)\right\rangle .
$$

The previous equation is similar to Eq. (1) except that now, $Z_{i}^{\alpha}$ and $A^{\alpha}$ represent the $z$ coordinates of the water oxygen sites and the area of the Voronoi polygons associated with the $\alpha=\mathrm{DS}$ and DTA headgroups, respectively.

The DTA and the DS curves present several common features with their counterparts shown in Fig. 4. In particular, DS headgroups are able to promote a larger extent of structuration in inner parts of the substrate, down to "depths" of $\sim 3-4$ water diameters. Note that the profile for these do- 
mains presents at least three well resolved and equally spaced peaks, while the one associated with DTA presents only two. In addition to the obvious differences in the charge distributions and eventual steric effects that determine the characteristics of each water-headgroup correlation functions at short distances, we are led to believe that the responses of the interface should also be affected by the local values of $\rho_{w}(z)$. We recall that DS exhibits less penetration into the water substrate, a fact that, in turn, would allow stronger responses of the interfacial water molecules to the Coulomb fields generated by its headgroups. On the other hand, the extent of the response of the water molecules in the closest solvation shells of DTA headgroups should necessarily be tempered by the stronger water-water interactions prevailing at deeper and denser aqueous substrates. Incidentally, we also point out the existence of a much smaller peak in the DTA profile located in the positive portion of the $z$ axis, which corresponds to a few water molecules bound to headgroups from outer positions.

A similar classification can be performed in the analysis of the local polarization fluctuations. In this case, the differences between positively and negatively charged headgroups are more dramatic. In the bottom panel of Fig. 5, we present results for polarization responses of the tessellated interface, namely,

$$
P_{\mu}^{\alpha}(z)=\frac{1}{A^{\alpha}}\left\langle\sum_{i} \cos \theta_{i}^{\alpha} \delta\left(Z_{i}^{\alpha}-Z_{\mathrm{HD}}^{\alpha}-z\right)\right\rangle,
$$

where

$$
\cos \theta_{i}^{\alpha}=\frac{\boldsymbol{\mu}_{i}^{\alpha} \cdot \hat{\mathbf{z}}}{\left|\boldsymbol{\mu}_{i}^{\alpha}\right|} .
$$

In the previous equation, the supraindex $\alpha$ refers to water dipoles lying in substrates of type $\alpha$. As expected, cationic and anionic headgroups promote dipolar alignments of opposite signs; however, the magnitude of the surface polarization response in the vicinity of DS groups is much more marked than the one observed in DTA domains. Although it is well known that the polarization response to external fields in bulk water is sign dependent, we are led to believe that the observed differences should have a similar origin to the one already mentioned when we examined density responses. To provide quantitative estimates, the areas of the main peaks of the two distributions differ in a factor of approximately 5: $\langle\cos \theta\rangle_{\mathrm{DS}} \sim 0.55$ and $\langle\cos \theta\rangle_{\mathrm{DTA}} \sim-0.09$.

\section{INTERFACE DYNAMICS}

In what follows, we will focus on several features related to the dynamics of the water substrate in close contact with the surfactant mixture. In this context, perhaps one basic question to be answered is how fast does an interfacial water molecule travel compared to the one located in the bulk. For clean interfaces, it is known that water molecules at liquid/ vapor interfaces diffuse between two and three times faster; moreover, the diffusion coefficient at the interface is inherently anisotropic, being the value along parallel directions $\left(D_{\|} \sim 0.8 \AA^{2} / \mathrm{ps}\right)$ somewhat larger than the one computed along perpendicular directions $\left(D_{\perp} \sim 0.5 \AA^{2} / \mathrm{ps}\right) .{ }^{30}$ For inter-
TABLE II. Dynamical parameters for the aqueous interface.

\begin{tabular}{lccc}
\hline \hline Zone & $\begin{array}{c}D_{\|} \\
\left(\AA^{2} / \mathrm{ps}\right)\end{array}$ & $\begin{array}{c}\tau_{\text {res }} \\
(\mathrm{ps})\end{array}$ & $\begin{array}{c}\tau_{\mu} \\
(\mathrm{ps})\end{array}$ \\
\hline I & 0.045 & 60 & $>\tau_{\text {res }}$ \\
II & 0.079 & 106 & 75 \\
III & 0.16 & 50 & 9.4 \\
"Bulk"a & 0.23 & $\cdots$ & $\cdots$ \\
\hline \hline
\end{tabular}

${ }^{\mathrm{a}}$ Computed at the center of the slab.

faces hosting surface active molecules with net charge, the scenario changes and conventional wisdom states that practically all dynamical modes of the substrate become significantly retarded. ${ }^{31}$

The usual strategy to analyze interfacial dynamics relies on the consideration of a series of regions parallel to the plane of the interface, with widths of the order of a few molecular diameters. ${ }^{32}$ This partition allows the tagging of water molecules according to their instantaneous locations. As such, one considers temporal correlations ascribed to these regions, whose implications remain physically sound, provided that the resulting characteristic decay time scales are much smaller than $\tau_{\text {res }}$ the average residence times of the molecules in each region. We pointed out in the previous section that, besides the overall interface width $\Delta z$ computed from the decay of $\rho_{w}(z)$ shown in Fig. 1, the interfacial landscape is modulated by local fluctuations promoted by the different degrees of penetration of the surfactant mixture into the substrate; moreover, we also found that the latter fluctuations prevail over lengthscales which are comparable to $\Delta z$. Under these circumstances, the proper identification of interfacial regions is not totally straightforward. Our criterion to define these regions was guided by the consideration of the profiles shown in Fig. 5 obtained from the tessellation procedure. More specifically, water molecules at the interface were discriminated according to their individual values of $\Delta Z_{i}=Z_{i}^{\alpha}-Z_{\mathrm{HD}}^{\alpha}$ as follows: (i) region I corresponded to water molecules in very low density environments $\left(\Delta Z_{i}>0\right)$; (ii) water molecules in region II corresponded to those included in the main peaks shown in the top panel of Fig. 5; (iii) finally, in region III, we included water molecules in the corresponding second solvation shells of the headgroups. With this definition, the resulting $\tau_{\text {res }}^{\alpha}$ for the three regions computed from the long time decays of the following correlation functions (not shown):

$$
C_{\text {sur }}^{\alpha}(t)=\frac{\left\langle\delta \eta_{i}^{\alpha}(0) \delta \eta_{i}^{\alpha}(t)\right\rangle}{\left\langle\left(\delta \eta_{i}^{\alpha}\right)^{2}\right\rangle}
$$

were of the order of $50-100 \mathrm{ps}$ for the three regions (see entries in Table II). In the previous equation, $\delta \mathcal{O}=\mathcal{O}-\langle\mathcal{O}\rangle$ and the characteristic function $\eta_{i}^{\alpha}(t)$ equals 1 if the $i$ th water molecule lies in region $\alpha$ at time $t$ and zero otherwise. ${ }^{29}$

For each region, estimates for the diffusion along directions parallel to the interface were obtained by computing the root mean square displacement along the $x y$ plane, namely, ${ }^{30}$ 


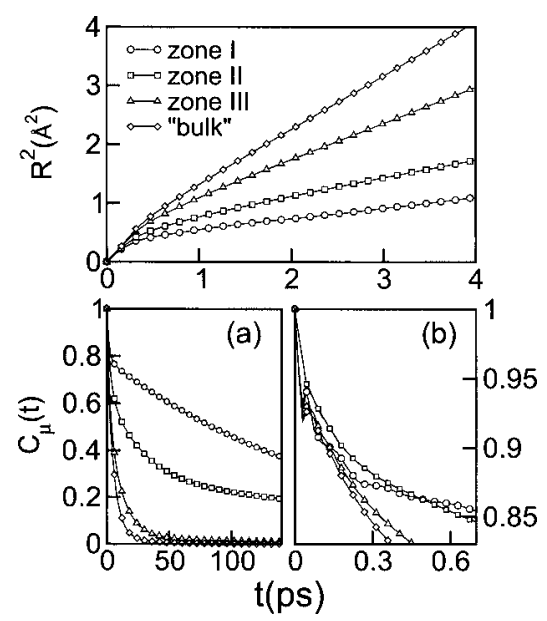

FIG. 6. Top panel: root mean square displacements for water molecules in different substrate layers (see text). Bottom panels: dipole-dipole autocorrelation functions for water molecules in different substrate layers. Long and short time behaviors are displayed in panels (a) and (b), respectively.

$$
D_{\|}^{\alpha} \sim \lim _{t \rightarrow \infty} \frac{R^{2}(t)}{4 t}
$$

where

$$
\begin{aligned}
R_{\alpha}^{2}\left(t-t^{\prime}\right)= & \left\langle\left[\left(x_{i}(t)-x_{i}\left(t^{\prime}\right)\right)^{2}+\left(y_{i}(t)-y_{i}\left(t^{\prime}\right)\right)^{2}\right]\right. \\
& \left.\times \eta_{i}^{\alpha}(t) \eta_{i}^{\alpha}\left(t^{\prime}\right)\right\rangle .
\end{aligned}
$$

The time dependence of the $R^{2}(t)$ for water lying at different regions is shown in the top panel of Fig. 6, while the corresponding results for $D_{\|}^{\alpha}$ appear in Table II. The general trend of the plots reveals gradual reductions of the translational dynamics as we approach the surfactant groups. Starting from a value of $D_{\|} \sim 0.15 \AA^{2} / \mathrm{ps}$ for region III (somewhat smaller than the one for bulk SPC/E, $D_{\text {bulk }}=0.25 \AA^{2} / \mathrm{ps}$ ), the water diffusion at the outer regions gradually diminishes to $D_{\|} \sim 0.08-0.04 \AA^{2} / \mathrm{ps}$. The slowness in the water displacements is the result of the strong coupling with the nearby headgroups, although the values of $D_{\|}$are still between three and four orders of magnitude larger than the lateral diffusion of the headgroups $\left(D_{\text {heads }} \approx 10^{-5} \AA^{2} / \mathrm{ps}\right)$. As a side remark, we would like to briefly comment on an attempt that we performed to get a rough appraisal of the perpendicular diffusion of water at the interface. At the most basic level of calculation, such an estimate could be inferred from the consideration of $\ell_{\alpha}$, the region widths, and the corresponding residence times, namely, $D_{\perp}^{\alpha} \sim \ell_{\alpha}^{2} / 2 \tau_{\text {res. }}^{\alpha}$. As found in the case of the bare interface, our simulation experiments yielded values of $D_{\perp}^{\alpha}$ which were comparable to those of $D_{\|}^{\alpha}$; however, we found that slight changes in the definition of the region boundaries would lead to non-negligible changes in the resulting values of $D_{\perp}^{\alpha}$. Given these circumstances, we did not proceed further and concluded that these crude estimates allowed us to establish neither conclusive trends in the magnitudes of $D_{\perp}^{\alpha}$ at different regions nor physically meaningful comparisons of the type $D_{\perp}^{\alpha}$ vs $D_{\|}^{\alpha}$.

Before closing the presentation of the results, we will briefly comment on orientational correlations of the water molecules within different regions of the substrate. In the bottom panel of Fig. 6, we show results for individual dipole-dipole time correlations functions,

$$
C \mu\left(t-t^{\prime}\right)=\frac{\left\langle\boldsymbol{\mu}_{i}^{\alpha}(t) \cdot \boldsymbol{\mu}_{i}^{\alpha}\left(t^{\prime}\right)\right\rangle}{\left|\boldsymbol{\mu}_{i}^{\alpha}\right|^{2}} .
$$

Estimates of the corresponding characteristic times $\tau_{\mu}$ expressed in terms of time integrals of $C \mu(t)$ also appear in Table II. The observed rotational dynamics is accordant with the previously described behavior of the translational modes, i.e., a clear tendency toward more retarded motions as we approach the headgroups from the bulk. Note, finally, that this trend does not appear in the initial, inertial period where the decay of the water molecules in the more external shell is the fastest (see inset in Fig. 6). As a possible explanation, we are led to believe that the latter feature might be associated with free librational motions of water molecules in low density environments which are likely to evolve much faster in outer regions than in deeper and denser regions of the water slab. As time progresses, these dynamical features should give place to rotational motions with a more marked diffusive character, modulated by much slower headgroup modes.

\section{CONCLUDING REMARKS}

The results presented in this paper extend our previous analysis on the characteristics of catanionic surfactants adsorbed at water/air interfaces to the full surface coverage regime. The general picture that emerges from both studies can be summarized as follows. The adsorption of catanionic surfactants leads to important modifications in the structure of an-originally bare-interface. The magnitude and characteristics of these modifications are very dependent of the degree of coverage. At low and midcoverages, the twodimensional density fields describing the adsorption of the surfactant species normally present large fluctuations, due to the strong Coulomb coupling between the different charged headgroups. The resulting structure of the detergents can be portrayed as clustered domains, with eventual percolative behavior, in which the hydrophobic tails remain practically in contact with the aqueous substrate. As such, the prevailing interactions determining the structure of these surfactant domains are those involving head-head and tail-substrate contributions. The presence of these surfactant "patches," in turn, also modify the local structure of the water substrate in close contact with the surfactant headgroups. The characteristics of these local modifications are dictated to a large extent by the different degree of penetration of cationic and anionic surfactant headgroups into the aqueous phase. Expressed in terms of the equilibrium positions of the headgroups, DTA detergents are localized approximately $1 \AA$ deeper into the substrate than DS ones.

At high surfactant coverages, the previous scenario changes at a qualitative level. Packing effects lead to more uniform density profiles for the surfactants and the aqueous domains as well. At a first glance, the structure of the surfactant mixture does not substantially differ from what one perceives in more conventional, one component ionic saturated monolayers. Tail-tail interactions become much more domi- 
nant and the observed tilts are close to $\theta_{\mathrm{tl}} \sim 45^{\circ}$. Similarly to the behavior observed at low surface coverages, DS and DTA exhibit differences in their corresponding locations with respect to the Gibbs dividing surface of the interface, although packing effects restrict the extent of the overall penetration of both headgroups which lie more externally with respect to the aqueous phase. As a direct consequence, the magnitude of the roughness induced in the underlying water substrate appears somewhat milder. Interestingly, an analysis based on a tessellation of the substrate reveals the presence of local domains all across the plane of the interface, associated with the locations of the surfactant headgroups; moreover, the magnitude of the local density and polarization fluctuations in DS domains look more enhanced than in DTA ones. This enhancement would be the direct consequence of the more external localization of the DS headgroups with respect to the plane of the interface. As such, as the local density of the substrate becomes smaller, the water molecules in close contact with DS headgroups would respond more "freely" to external Coulomb fields than those located in deeper layers of the substrate.

Finally, and from the dynamical side, we have computed estimates for the diffusion coefficients of water molecules adjacent to the interface. At full coverages, and as we move from the bulk into the interfacial region, there seems to be a global reduction in the diffusion along parallel directions to the interface plane by factor of $\sim 4-6$. This retardation in the diffusion is akin to the well documented dynamical behavior observed in "trapped" water in the vicinity of complex biological molecules, although our estimates for $D_{\|}$are still not comparable to the diffusive behavior of the surfactants (which are between two and three orders of magnitude slower). Similar general trends were also observed in the rotational modes of the water molecules lying in close contact with the surfactants.

\section{ACKNOWLEDGMENTS}

Two of the authors (J.R. and D.L.) are staff members of CONICET (Argentina).

\footnotetext{
${ }^{1}$ See, for example, Mixed Surfactant Systems, edited by K. Ogino and A. Mashajido (Dekker, New York, 1993); Phenomena in Mixed Surfactant Systems, edited by J. F. Scamerhorn (ACS, Washington, DC, 1986).

${ }^{2}$ P. Jokela, B. Jönsson, A. S. Sadaghiani, and A. Khan, Langmuir 7, 889 (1991); A. Khan and E. Marques, in Specialists Surfactants, edited by I. D. Robb (Chapman and Hall, London, 1997).

${ }^{3}$ See E. U. Kaler, K. L. Herrington, D. D. Miller, and J. A. N. Zasadzinski, in Structure and Dynamics of Strongly Interacting Colloids and Supramolecular Aggregates in Solution, edited by S. H. Chen, J. S. Huang, and P. Tartaglia (Kluwer Academic, Dordrecht, 1992), pp. 571-577.
}

${ }^{4}$ E. W. Kaler, A. K. Murthy, B. Rodriguez, and J. A. N. Zasadzinski, Science 245, 1371 (1989).

${ }^{5}$ E. W. Kaler, K. L. Herrington, A. K. Murthy, and J. A. N. Zasadzinski, J. Phys. Chem. 96, 6698 (1992); K. L. Herrington, E. W. Kaler, D. D. Miller, J. A. Zasadzinski, and S. Chiruvolu, ibid. 97, 13792 (1993).

${ }^{6}$ T. Zemb, M. Dubois, B. Demé, and T. Gulik-Kryzwicki, Science 283, 816 (1999); M. Dubois, B. Dermé, T. Gulik-Kryzwicki, J.-C. Dedieu, C. Vautrin, S. Désert, E. Perez, and T. Zemb, Nature (London) 411, 672 (2001).

${ }^{7}$ C. Tondre and C. Caillet, Adv. Colloid Interface Sci. 93, 115 (2001).

${ }^{8}$ H. W. Hoyer, A. Marmo, and M. Zoellner, J. Phys. Chem. 65, 1804 (1961).

${ }^{9}$ J. M. Corkill, J. F. Goodman, C. P. Ogden, and J. R. Tate, Proc. R. Soc. London, Ser. A 273, 83 (1963).

${ }^{10}$ H. Matsuki, M. Aratono, S. Kaneshina, and K. Motomura, J. Colloid Interface Sci. 191, 120 (1997); J. B. Huang and G. X. Zhao, Colloid Polym. Sci. 273, 156 (1995).

${ }^{11}$ J. Rodakiewicz-Novak, J. Colloid Interface Sci. 85, 586 (1982).

${ }^{12}$ I. Varga, T. Keszthelyi, R. Mészáros, O. Hakkel, and T. Gilányi, J. Phys. Chem. B 109, 872 (2005).

${ }^{13}$ T. Gilányi, R. Mészáros, and I. Varga, Langmuir 16, 3200 (2000).

${ }^{14}$ M. I. Viseu, A. Goncalvez da Silva, and S. M. B. Costa, Langmuir 17, 1529 (2001)

${ }^{15}$ Y. Wang, C. M. Pereira, E. F. Marques, R. O. Brito, E. S. Ferreira, and F. Silva, Thin Solid Films 515, 2031 (2006).

${ }^{16}$ D. Góralczyk, J. Clim. 179, 211 (1996); D. Góralczyk, Colloids Surf., A 196, 25 (2002).

${ }^{17}$ M. Bergstrom and J. C. Eriksson, Langmuir 16, 7173 (2000).

${ }^{18}$ J. Rodakiewicz-Nowak, J. Colloid Interface Sci. 84, 532 (1981).

${ }^{19}$ N. Filipović-Vinceković, I. Juranović, and Ž. Grhek, Colloids Surf., A 125, 115 (1997).

${ }^{20}$ H. Dominguez, J. Phys. Chem. B 106, 5915 (2002).

${ }^{21}$ H. Dominguez, J. Clim. 274, 665 (2004).

${ }^{22}$ H. Dominguez and M. Rivera, Langmuir 21, 7257 (2005).

${ }^{23}$ J. Rodriguez, E. Clavero, and D. Laria, J. Phys. Chem. B 109, 24427 (2005).

${ }^{24}$ J. S. Rowlinson and B. Widom, Molecular Theory of Capilarity (Clarendon, Oxford, 1982).

${ }^{25}$ D. Dragcević, M. Bujan, Ž. Grhek, and N. Filopović-Vicenković, Colloid Polym. Sci. 273, 967 (1995).

${ }^{26}$ J. Eastoe, J. Dalton, J. Rogueda, D. Sharpe, J. Dong, and J. R. P. Webster, Langmuir 12, 2706 (1996).

${ }^{27}$ R. S. Taylor, L. X. Dang, and B. C. Garrett, J. Phys. Chem. 100, 11720 (1996).

${ }^{28}$ S. A. Pandit, D. Bostick, and M. L. Berkowitz, J. Chem. Phys. 119, 2199 (2003).

${ }^{29}$ In fact, $\left\langle\eta_{i}^{\alpha}\right\rangle$ scales as the ratio between the volume of the slab and the volume of the system. In macroscopic systems, this ratio is of the order of $\mathcal{O}\left(N_{w}^{-1}\right)$, so for all practical purposes this average can be approximated by zero.

${ }^{30}$ A rigorous analysis of diffusive processes at interfaces shows that results from Eq. (14) are not fully satisfactory and should only be taken as estimates. For a general discussion, see P. Liu, E. Harder, and B. J. Berne, J. Phys. Chem. B 108, 6595 (2004).

${ }^{31}$ N. Nandi, K. Bhattacharyya, and B. Bagchi, Chem. Rev. (Washington, D.C.) 100, 2013 (2000).

${ }^{32}$ See, for example, I. Benjamin, J. Chem. Phys. 97, 1432 (1992); R. M. Townsend and S. A. Rice, ibid. 94, 2207 (1991); S. H. Lee and P. J. Rossky, ibid. 100, 3334 (1994); J. Chanda and S. Bandyopadhyay, J. Phys. Chem. B 110, 23482 (2006). 